\title{
II. CARBON AND CARBONATE ANALYSES, LEG 33
}

Donald H. Cameron, Scripps Institution of Oceanography, La Jolla, California

Leg 33 carbonate samples were collected onboard ship in 3-cc vials at the time the core was split open. On shore the samples were dried and ground to a homogeneous powder. The ground sediment was redried at $105^{\circ}-110^{\circ} \mathrm{C}$ and two samples from each vial, a $0.1-\mathrm{g}$ and a 0.5-g sample, were then weighted into LECO crucibles. The $0.5-\mathrm{g}$ sample was acidified with dilute hydrochloric acid, washed with distilled water, redried, and analyzed for acid insoluble (organic) carbon using a LECO 70 carbon analyzer. The 0.1 -g sample was treated only with distilled water to cake the sample before analysis for total carbon. If the result showed less than $10 \% \mathrm{CaCO}_{3}$, an additional $0.5-\mathrm{g}$ sample was analyzed for greater accuracy. The calcium carbonate percentage was calculated as follows: (\% total C - \% organic C) $\times$ $8.33=\% \mathrm{CaCO}_{3}$. Although other carbonates may be present, all acid-soluble carbon was calculated as calcium carbonate. All results are given in weight percent.

Detailed descriptions of technique and theory are in Bader, Gerard, et al., 1970.

For control purposes a standard sediment was made up from Deep Sea Drilling material and analyzed for total carbon at predetermined intervals with regular samples. Listed below are the statistical data for this standard.

\begin{tabular}{ccccc}
\hline $\begin{array}{c}\text { DSDP } \\
\text { Std. }\end{array}$ & $\begin{array}{c}\text { No. of } \\
\text { Samples }\end{array}$ & $\begin{array}{c}\text { Total Carbon } \\
\text { as \% } \mathrm{CaCO}_{3}\end{array}$ & $\begin{array}{c}\text { Standard } \\
\text { Deviation }\end{array}$ & $\begin{array}{c}\text { Maximum } \\
\text { Range }\end{array}$ \\
\hline 2 & 53 & 80.7 & $0.8 \%$ & $4.0 \%$ \\
\hline
\end{tabular}

These data indicate the precision of the mechanical aspect of the LECO analysis and do not necessarily reflect the precision of the total analystical procedure, which may be affected by factors such as inhomogeneous standard or contamination during sample preparation.

\section{REFERENCE}

Bader, R.G., Gerard, R.D., et al., 1970. Initial Reports of the Deep Sea Drilling Project, Volume 4: Washington (U.S. Government Printing Office).

TABLE 1

Carbon-Carbonate Analyses

\begin{tabular}{lcccc}
\hline $\begin{array}{c}\text { Core-Section, } \\
\text { Top of Interval } \\
(\mathrm{cm})\end{array}$ & $\begin{array}{c}\text { Subbottom } \\
\text { Depth } \\
(\mathrm{m})\end{array}$ & $\begin{array}{c}\text { Total } \\
\text { Carbon } \\
(\%)\end{array}$ & $\begin{array}{c}\text { Organic } \\
\text { Carbon }\end{array}$ & $\mathrm{CaCO}_{3}$ \\
\hline Site 314 & & & & \\
$3-7,0$ & 44.0 & 1.0 & 0.7 & 3 \\
$3-7,0$ & 44.0 & 2.6 & 0.0 & 21 \\
Hole 315 & & & & \\
$1-1,119$ & 1.2 & 11.3 & 0.0 & 94 \\
\hline
\end{tabular}

TABLE 1 - Continued

\begin{tabular}{|c|c|c|c|c|}
\hline $\begin{array}{l}\text { Core-Section, } \\
\text { Top of Interval } \\
\text { (cm) }\end{array}$ & $\begin{array}{l}\text { Subbottom } \\
\text { Depth } \\
\text { (m) }\end{array}$ & $\begin{array}{l}\text { Total } \\
\text { Carbon } \\
(\%)\end{array}$ & $\begin{array}{l}\text { Organic } \\
\text { Carbon }\end{array}$ & $\mathrm{CaCO}_{3}$ \\
\hline $1-2,28$ & 1.8 & 8.2 & 0.1 & 67 \\
\hline $1-2,86$ & 2.4 & 11.3 & 0.0 & 94 \\
\hline $1-2,118$ & 2.7 & 10.5 & 0.1 & 87 \\
\hline $1-3,79$ & 3.8 & 11.1 & 0.0 & 92 \\
\hline $1-4,44$ & 4.9 & 10.5 & 0.0 & 87 \\
\hline $1-5,20$ & 6.2 & 11.3 & 0.0 & 94 \\
\hline $1-6,135$ & 8.9 & 10.5 & 0.1 & 87 \\
\hline $4-2,116$ & 59.2 & 10.6 & 0.0 & 88 \\
\hline $4-2,134$ & 59.3 & 9.0 & 0.0 & 75 \\
\hline $4-3,50$ & 60.0 & 10.2 & 0.1 & 85 \\
\hline $4-3,87$ & 60.4 & 11.0 & 0.0 & 92 \\
\hline $4-4,90$ & 61.9 & 8.4 & 0.0 & 69 \\
\hline $4-4,120$ & 62.2 & 10.5 & 0.0 & 87 \\
\hline $4-5,3$ & 62.5 & 9.1 & 0.0 & 75 \\
\hline $4-5,140$ & 63.9 & 11.4 & 0.0 & 95 \\
\hline $4-6,17$ & 64.2 & 11.1 & 0.0 & 92 \\
\hline $4-7,0$ & 65.5 & 11.0 & 0.0 & 91 \\
\hline \multicolumn{5}{|l|}{ Hole $315 \mathrm{~A}$} \\
\hline $1-1,100$ & 77.0 & 11.6 & 0.0 & 96 \\
\hline $1-2,20$ & 77.7 & 10.8 & 0.0 & 90 \\
\hline $1-3,20$ & 79.2 & 10.8 & 0.0 & 90 \\
\hline $1-4,45$ & 81.0 & 10.6 & 0.0 & 88 \\
\hline $1-5,30$ & 82.3 & 11.1 & 0.0 & 93 \\
\hline $1-5,130$ & 83.3 & 10.6 & 0.0 & 88 \\
\hline $1-6,131$ & 84.8 & 11.2 & 0.0 & 93 \\
\hline $2-1,81$ & 124.3 & 10.7 & 0.0 & 89 \\
\hline $3-1,60$ & 142.8 & 10.6 & 0.0 & 88 \\
\hline $3-3,10$ & 145.3 & 10.9 & 0.0 & 91 \\
\hline $3-4,70$ & 147.4 & 11.1 & 0.0 & 92 \\
\hline $3-5,1$ & 148.2 & 10.0 & 0.0 & 83 \\
\hline $3-6,33$ & 150.0 & 10.3 & 0.0 & 85 \\
\hline $4-1,124$ & 257.2 & 11.0 & 0.0 & 91 \\
\hline $4-2,51$ & 258.0 & 11.1 & 0.0 & 92 \\
\hline $4-3,20$ & 259.2 & 11.3 & 0.0 & 94 \\
\hline $4-3,100$ & 260.0 & 11.1 & 0.0 & 92 \\
\hline $4-3,120$ & 260.2 & 11.5 & 0.0 & 96 \\
\hline $5-1,8$ & 370.1 & 11.1 & 0.0 & 92 \\
\hline $5-1,66$ & 370.7 & 10.7 & 0.0 & 89 \\
\hline $5-2,95$ & 372.5 & 10.0 & 0.0 & 83 \\
\hline $6-1,61$ & 465.6 & 11.0 & 0.0 & 91 \\
\hline $6-2,50$ & 467.0 & 11.1 & 0.0 & 92 \\
\hline $6-3,17$ & 468.2 & 10.6 & 0.0 & 88 \\
\hline $6-7,0$ & 474.0 & 10.8 & 0.0 & 90 \\
\hline $7-1,118$ & 513.7 & 11.5 & 0.0 & 95 \\
\hline $7-2,86$ & 514.9 & 11.1 & 0.0 & 92 \\
\hline $8-1,21$ & 588.7 & 11.4 & 0.0 & 94 \\
\hline $8-2,75$ & 590.8 & 11.4 & 0.0 & 95 \\
\hline $8-3,30$ & 591.8 & 11.4 & 0.0 & 95 \\
\hline $9-1,112$ & 703.6 & 11.6 & 0.0 & 96 \\
\hline $9-2,15$ & 704.2 & 11.5 & 0.0 & 96 \\
\hline $10-1,13$ & 730.9 & 10.8 & 0.0 & 90 \\
\hline $10-2,45$ & 732.8 & 11.0 & 0.0 & 91 \\
\hline $10-3,90$ & 734.7 & 11.2 & 0.0 & 93 \\
\hline $10-4,0$ & 735.3 & 11.4 & 0.0 & 95 \\
\hline $10-4,85$ & 736.2 & 2.7 & 0.1 & 22 \\
\hline $10-5,8$ & 736.9 & 1.1 & 0.0 & 9 \\
\hline $10-5,140$ & 738.2 & 0.1 & 0.0 & 1 \\
\hline $10-6,88$ & 739.2 & 1.0 & 0.0 & 9 \\
\hline
\end{tabular}


TABLE 1 - Continued

\begin{tabular}{|c|c|c|c|c|}
\hline $\begin{array}{l}\text { Core-Section, } \\
\text { Top of Interval } \\
(\mathrm{cm})\end{array}$ & $\begin{array}{l}\text { Subbottom } \\
\text { Depth } \\
\text { (m) }\end{array}$ & $\begin{array}{c}\text { Total } \\
\text { Carbon } \\
(\%)\end{array}$ & $\begin{array}{l}\text { Organic } \\
\text { Carbon }\end{array}$ & $\mathrm{CaCO}_{3}$ \\
\hline $10-7,0$ & 739.8 & 3.4 & 0.0 & 28 \\
\hline $15-1,120$ & 779.2 & 0.3 & 0.0 & 3 \\
\hline $15-2,39$ & 779.9 & 0.2 & 0.0 & 1 \\
\hline $16-1,98$ & 788.5 & 0.1 & 0.0 & 1 \\
\hline $16-2,14$ & 789.1 & 0.1 & 0.0 & 1 \\
\hline $16-2,117$ & 790.2 & 1.8 & 0.1 & 15 \\
\hline $17-1,117$ & 798.2 & 11.8 & 0.0 & 98 \\
\hline $17-2,70$ & 799.2 & 11.8 & 0.1 & 98 \\
\hline $17-2,85$ & 799.4 & 1.8 & 0.0 & 15 \\
\hline $18-1,54$ & 807.0 & 4.6 & 0.0 & 38 \\
\hline $18-1,86$ & 807.4 & 11.7 & 0.0 & 97 \\
\hline $18-2,71$ & 808.7 & 6.1 & 0.0 & 51 \\
\hline $18-4,145$ & 812.5 & 5.6 & 0.0 & 46 \\
\hline $19-2,118$ & 818.7 & 7.4 & 0.0 & 62 \\
\hline $19-3,52$ & 819.5 & 4.5 & 0.1 & 37 \\
\hline $19-3,149$ & 820.5 & 5.8 & 0.0 & 48 \\
\hline $19-4,48$ & 821.0 & 6.8 & 0.0 & 57 \\
\hline $19-4,120$ & 821.7 & 7.1 & 0.1 & 58 \\
\hline $19-5,29$ & 822.3 & 6.1 & 0.0 & 51 \\
\hline $19-6,62$ & 824.1 & 5.1 & 0.1 & 41 \\
\hline $20-1,143$ & 826.9 & 5.2 & 0.0 & 43 \\
\hline $20-2,96$ & 828.0 & 7.5 & 0.1 & 62 \\
\hline $20-3,53$ & 829.0 & 4.3 & 0.0 & 35 \\
\hline $20-4,41$ & 831.4 & 5.9 & 0.0 & 49 \\
\hline $20-4,139$ & 830.4 & 8.7 & 0.0 & 72 \\
\hline $20-5,78$ & 832.3 & 4.2 & 0.1 & 34 \\
\hline $21-2,32$ & 836.8 & 10.8 & 0.0 & 90 \\
\hline $21-2,46$ & 837.0 & 6.2 & 0.1 & 51 \\
\hline $21-5,90$ & 841.9 & 4.7 & 0.0 & 39 \\
\hline $22-3,75$ & 848.3 & 6.3 & 0.1 & 52 \\
\hline $23-2,1$ & 855.5 & 3.4 & 0.1 & 28 \\
\hline $24-1,83$ & 873.8 & 1.7 & 0.1 & 14 \\
\hline $24-2,30$ & 874.8 & 8.5 & 0.0 & 70 \\
\hline $24-3,112$ & 877.1 & 9.1 & 0.0 & 76 \\
\hline $24-6,26$ & 880.8 & 6.6 & 0.0 & 55 \\
\hline $25-1,136$ & 893.4 & 4.1 & 0.0 & 34 \\
\hline $25-2,72$ & 894.2 & 5.5 & 0.0 & 45 \\
\hline $25-3,48$ & 895.5 & 2.6 & 0.0 & 21 \\
\hline $26-1,130$ & 912.3 & 1.0 & 0.0 & 8 \\
\hline $26-2,36$ & 912.9 & 0.3 & 0.1 & 1 \\
\hline $27-1,122$ & 931.2 & 0.1 & 0.0 & 0 \\
\hline $27-6,62$ & 938.1 & 0.1 & 0.0 & 0 \\
\hline $28-2,1$ & 952.8 & 0.2 & 0.1 & 1 \\
\hline $28-3,76$ & 950.5 & 0.1 & 0.0 & 1 \\
\hline $29-1,55$ & 968.6 & 0.1 & 0.1 & 1 \\
\hline $29-2,14$ & 969.6 & 0.1 & 0.1 & 1 \\
\hline $30-1,100$ & 988.0 & 0.6 & 0.1 & 4 \\
\hline $30-2,40$ & 988.9 & 0.2 & 0.0 & 1 \\
\hline $30-2,135$ & 989.9 & 0.1 & 0.0 & 0 \\
\hline \multicolumn{5}{|l|}{ Site 316} \\
\hline $1-1,25$ & 0.3 & 10.9 & 0.1 & 91 \\
\hline $1-1,33$ & 0.3 & 7.6 & 0.1 & 62 \\
\hline $1-2,85$ & 2.4 & 7.0 & 0.1 & 58 \\
\hline $1-3,103$ & 4.0 & 11.3 & 0.0 & 94 \\
\hline $1-4,57$ & 5.1 & 10.9 & 0.0 & 91 \\
\hline $1-5,75$ & 6.8 & 11.2 & 0.0 & 93 \\
\hline $1-6,40$ & 7.9 & 11.4 & 0.0 & 94 \\
\hline $2-1,104$ & 154.0 & 10.4 & 0.0 & 86 \\
\hline $2-2,75$ & 155.3 & 10.5 & 0.0 & 87 \\
\hline $3-1,124$ & 268.2 & 10.3 & 0.0 & 86 \\
\hline $3-2,75$ & 269.3 & 10.3 & 0.0 & 86 \\
\hline $4-1,122$ & 391.7 & 10.0 & 0.0 & 83 \\
\hline $4-2,131$ & 393.3 & 9.1 & 0.0 & 76 \\
\hline $5-1,111$ & 448.6 & 11.2 & 0.0 & 93 \\
\hline $5-2,103$ & 450.0 & 10.9 & 0.0 & 91 \\
\hline
\end{tabular}

TABLE 1 - Continued

\begin{tabular}{|c|c|c|c|c|}
\hline $\begin{array}{l}\text { Core-Section, } \\
\text { Top of Interval } \\
\text { (cm) }\end{array}$ & $\begin{array}{l}\text { Subbottom } \\
\text { Depth } \\
\text { (m) }\end{array}$ & $\begin{array}{c}\text { Total } \\
\text { Carbon } \\
(\%)\end{array}$ & $\begin{array}{l}\text { Organic } \\
\text { Carbon }\end{array}$ & $\mathrm{CaCO}_{3}$ \\
\hline $7-1,144$ & 467.9 & 11.4 & 0.1 & 94 \\
\hline $7-2,5$ & 468.1 & 12.2 & 0.0 & 101 \\
\hline $8-1,141$ & 477.4 & 10.7 & 0.0 & 89 \\
\hline $9-1,100$ & 486.5 & 11.2 & 0.0 & 93 \\
\hline $10-1,91$ & 495.9 & 5.8 & 0.0 & 48 \\
\hline $11-1,135$ & 515.4 & 11.2 & 0.0 & 93 \\
\hline $12-1,123$ & 524.7 & 11.6 & 0.0 & 97 \\
\hline $12-2,15$ & 525.2 & 10.7 & 0.0 & 89 \\
\hline $13-1,54$ & 533.5 & 10.8 & 0.0 & 90 \\
\hline $14-1,88$ & 543.4 & 9.1 & 0.0 & 75 \\
\hline $15-1,101$ & 553.0 & 8.9 & 0.0 & 74 \\
\hline $16-1,137$ & 562.9 & 10.5 & 0.0 & 87 \\
\hline $16-2,122$ & 564.2 & 7.6 & 0.0 & 63 \\
\hline $17-1,83$ & 571.8 & 11.6 & 0.0 & 96 \\
\hline $17-2,104$ & 573.5 & 11.5 & 0.0 & 96 \\
\hline $18-1,110$ & 581.6 & 5.9 & 0.0 & 49 \\
\hline $18-2,102$ & 583.0 & 6.6 & 0.0 & 55 \\
\hline $18-3,117$ & 584.7 & 9.3 & 0.0 & 78 \\
\hline $18-4,48$ & 585.5 & 11.0 & 0.0 & 91 \\
\hline $18-5,83$ & 587.3 & 11.9 & 0.0 & 99 \\
\hline $19-1,89$ & 609.9 & 5.7 & 0.0 & 47 \\
\hline $19-2,73$ & 611.2 & 10.8 & 0.0 & 90 \\
\hline $19-3,59$ & 612.6 & 10.7 & 0.0 & 89 \\
\hline $19-4,10$ & 613.6 & 10.7 & 0.0 & 89 \\
\hline $20-1,102$ & 629.0 & 9.1 & 0.0 & 75 \\
\hline $20-2,112$ & 630.6 & 9.2 & 0.0 & 77 \\
\hline $20-3,49$ & 631.5 & 10.8 & 0.0 & 89 \\
\hline $20-4,31$ & 632.8 & 9.2 & 0.0 & 76 \\
\hline $21-2,46$ & 646.0 & 9.9 & 0.0 & 82 \\
\hline $21-3,23$ & 639.5 & 7.1 & 0.1 & 58 \\
\hline $21-4,9$ & 640.7 & 10.3 & 0.1 & 85 \\
\hline $21-6,96$ & 642.1 & 6.5 & 0.4 & 52 \\
\hline $22-1,116$ & 648.2 & 8.1 & 0.0 & 68 \\
\hline $22-2,12$ & 648.6 & 10.7 & 0.0 & 89 \\
\hline $22-3,54$ & 650.5 & 8.7 & 0.0 & 73 \\
\hline $23-1,104$ & 667.0 & 10.1 & 0.0 & 84 \\
\hline $23-2,54$ & 668.0 & 9.4 & 0.0 & 78 \\
\hline $23-3,97$ & 670.0 & 9.5 & 0.1 & 78 \\
\hline $23-4,67$ & 671.2 & 9.9 & 0.0 & 82 \\
\hline $23-5,36$ & 672.4 & 10.5 & 0.0 & 87 \\
\hline $24-1,89$ & 685.9 & 10.6 & 0.0 & 88 \\
\hline $24-2,20$ & 686.7 & 10.9 & 0.0 & 91 \\
\hline $24-3,63$ & 688.6 & 11.0 & 0.0 & 91 \\
\hline $24-4,43$ & 689.9 & 10.1 & 0.0 & 84 \\
\hline $24-5,102$ & 692.0 & 9.9 & 0.0 & 82 \\
\hline $25-1,45$ & 704.5 & 7.2 & 0.0 & 60 \\
\hline $25-2,20$ & 705.7 & 5.5 & 0.0 & 46 \\
\hline $25-4,101$ & 709.5 & 11.1 & 0.0 & 93 \\
\hline $25-5,61$ & 710.6 & 9.8 & 0.0 & 81 \\
\hline $26-1,60$ & 723.6 & 9.1 & 0.0 & 76 \\
\hline $26-1,90$ & 723.9 & 5.2 & 0.0 & 43 \\
\hline $26-3,100$ & 730.3 & 10.4 & 0.0 & 86 \\
\hline $26-5,130$ & 727.0 & 6.4 & 0.1 & 52 \\
\hline $27-1,142$ & 743.4 & 2.3 & 0.0 & 19 \\
\hline $27-2,138$ & 744.9 & 6.1 & 0.0 & 51 \\
\hline $27-4,103$ & 747.5 & 2.1 & 0.0 & 17 \\
\hline $28-1,64$ & 771.1 & 5.0 & 0.0 & 42 \\
\hline $28-7,0$ & 779.5 & 0.3 & 0.0 & 3 \\
\hline $30-2,132$ & 830.3 & 10.5 & 0.0 & 87 \\
\hline \multicolumn{5}{|l|}{ Hole 317} \\
\hline $1-1,16$ & 0.7 & 11.6 & 0.0 & 96 \\
\hline $1-1,120$ & 1.7 & 11.5 & 0.0 & 96 \\
\hline $1-2,58$ & 2.6 & 11.5 & 0.0 & 96 \\
\hline $1-3,64$ & 4.1 & 11.6 & 0.0 & 96 \\
\hline $1-4,100$ & 6.0 & 11.5 & 0.0 & 95 \\
\hline
\end{tabular}


TABLE 1 - Continued

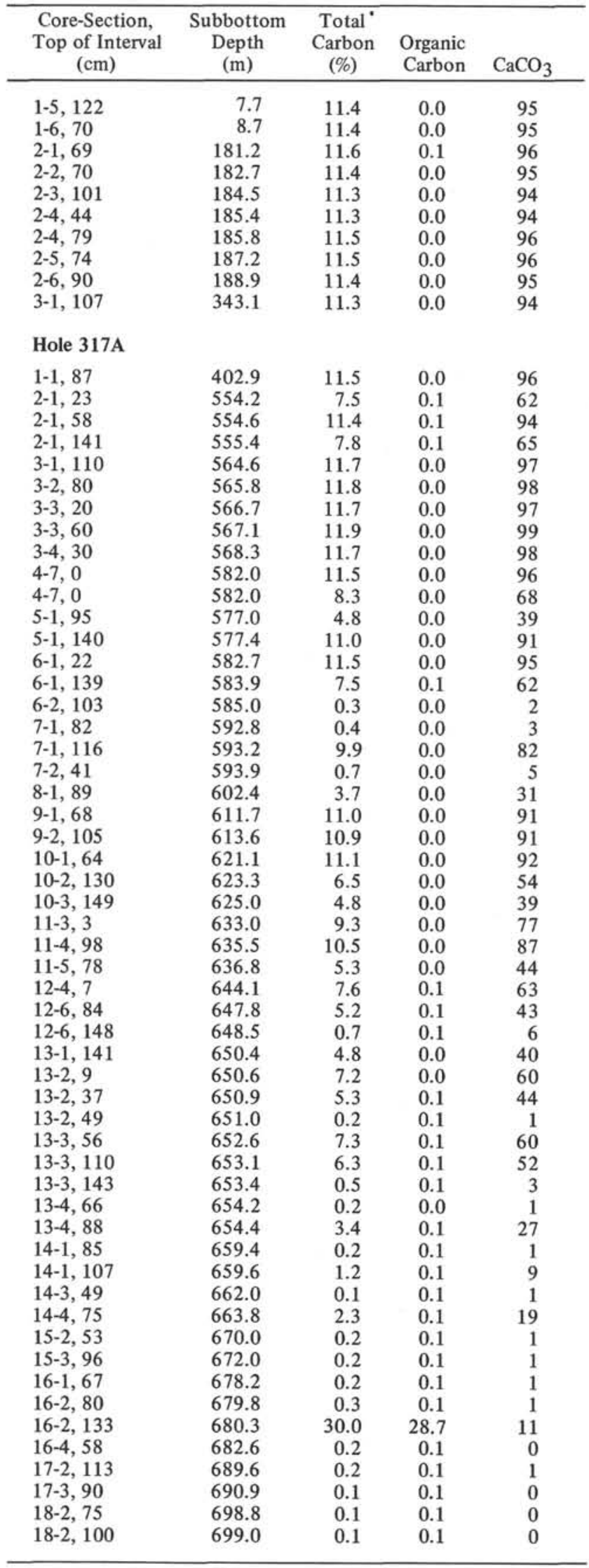

TABLE 1 - Continued

\begin{tabular}{lcccc}
\hline $\begin{array}{c}\text { Core-Section } \\
\text { Top of Interval } \\
(\mathrm{cm})\end{array}$ & $\begin{array}{c}\text { Subbottom } \\
\text { Depth } \\
(\mathrm{m})\end{array}$ & $\begin{array}{c}\text { Total } \\
\text { Carbon } \\
(\%)\end{array}$ & $\begin{array}{c}\text { Organic } \\
\text { Carbon }\end{array}$ & $\mathrm{CaCO}_{3}$ \\
\hline $19-3,58$ & 719.1 & 0.2 & 0.1 & 0 \\
$19-3,105$ & 719.6 & 0.1 & 0.1 & 0 \\
$19-4,83$ & 720.8 & 0.1 & 0.1 & 0 \\
$20-2,50$ & 727.0 & 0.3 & 0.1 & 1 \\
$21-3,91$ & 738.4 & 0.2 & 0.1 & 0 \\
$21-4,137$ & 740.4 & 0.4 & 0.1 & 2 \\
$22-2,91$ & 755.9 & 0.1 & 0.1 & 0 \\
$22-4,7$ & 758.1 & 0.1 & 0.1 & 0 \\
$22-4,63$ & 758.6 & 0.1 & 0.1 & 0 \\
$22-4,139$ & 759.4 & 0.1 & 0.1 & 0 \\
$23-1,127$ & 764.3 & 0.2 & 0.1 & 0 \\
$24-1,67$ & 773.2 & 0.1 & 0.1 & 0 \\
$24-2,109$ & 775.1 & 0.1 & 0.1 & 0 \\
$24-4,65$ & 777.7 & 0.2 & 0.1 & 0 \\
$24-4,115$ & 778.2 & 0.2 & 0.1 & 0 \\
$25-2,60$ & 793.6 & 0.1 & 0.1 & 0 \\
$25-5,39$ & 797.9 & 0.1 & 0.1 & 0 \\
$26-1,107$ & 821.1 & 0.1 & 0.1 & 0 \\
$26-3,100$ & 824.0 & 0.1 & 0.1 & 0 \\
$26-4,98$ & 825.5 & 0.1 & 0.1 & 0 \\
$26-5,149$ & 827.5 & 0.1 & 0.1 & 0 \\
$27-5,140$ & 837.4 & 0.1 & 0.1 & 1 \\
$28-1,120$ & 849.7 & 0.1 & 0.1 & 0 \\
$28-2,34$ & 850.3 & 0.1 & 0.1 & 0 \\
$28-6,102$ & 857.0 & 0.2 & 0.1 & 1 \\
$29-2,140$ & 870.4 & 0.1 & 0.1 & 0 \\
$29-5,17$ & 873.7 & 0.1 & 0.1 & 0 \\
$30-1,88$ & 887.4 & 0.2 & 0.1 & 0 \\
$30-2,26$ & 888.3 & 0.1 & 0.1 & 0 \\
& & & &
\end{tabular}

Hole 317B

\begin{tabular}{|c|c|c|c|c|}
\hline $1-1,30$ & 0.4 & 11.5 & 0.1 & 95 \\
\hline $1-2,40$ & 2.0 & 11.7 & 0.1 & 97 \\
\hline $1-6,106$ & 8.7 & 11.6 & 0.1 & 96 \\
\hline $2-1,66$ & 7.2 & 11.7 & 0.0 & 97 \\
\hline $2-1,86$ & 7.4 & 11.7 & 0.0 & 97 \\
\hline $2-3,90$ & 10.4 & 11.7 & 0.0 & 97 \\
\hline $2-4,22$ & 11.2 & 11.7 & 0.0 & 97 \\
\hline $2-4,135$ & 12.4 & 11.8 & 0.0 & 98 \\
\hline $3-1,36$ & 16.4 & 11.8 & 0.1 & 98 \\
\hline $3-4,50$ & 21.0 & 11.7 & 0.1 & 97 \\
\hline $3-5,56$ & 22.6 & 11.7 & 0.1 & 97 \\
\hline $4-2,46$ & 27.5 & 11.7 & 0.1 & 97 \\
\hline $4-3,83$ & 29.3 & 11.8 & 0.1 & 98 \\
\hline $5-1,85$ & 35.9 & 11.5 & 0.1 & 96 \\
\hline $5-4,66$ & 40.2 & 11.7 & 0.0 & 97 \\
\hline $5-6,22$ & 42.7 & 11.7 & 0.0 & 97 \\
\hline $6-3,75$ & 48.3 & 11.5 & 0.0 & 95 \\
\hline $6-5,56$ & 51.1 & 11.8 & 0.0 & 98 \\
\hline $7-4,61$ & 59.1 & 11.8 & 0.0 & 98 \\
\hline $8-2,76$ & 65.8 & 11.9 & 0.0 & 99 \\
\hline $8-4,66$ & 68.7 & 11.9 & 0.0 & 99 \\
\hline $9-1,66$ & 73.7 & 11.7 & 0.0 & 97 \\
\hline $9-3,75$ & 76.8 & 11.7 & 0.0 & 97 \\
\hline $9-5,90$ & 79.9 & 11.6 & 0.1 & 97 \\
\hline $10-1,84$ & 83.3 & 11.7 & 0.1 & 96 \\
\hline $10-3,65$ & 86.2 & 11.7 & 0.0 & 97 \\
\hline $11-2,135$ & 94.9 & 11.6 & 0.0 & 97 \\
\hline $12-1,75$ & 102.3 & 11.6 & 0.0 & 96 \\
\hline $12-3,45$ & 105.0 & 11.5 & 0.0 & 96 \\
\hline $12-5,65$ & 108.2 & 11.7 & 0.0 & 97 \\
\hline $13-1,129$ & 112.8 & 11.7 & 0.0 & 97 \\
\hline $13-3,66$ & 115.2 & 11.7 & 0.0 & 97 \\
\hline $16-1,139$ & 141.2 & 11.7 & 0.0 & 97 \\
\hline $16-2,65$ & 142.0 & 10.5 & 0.0 & 87 \\
\hline $16-4,105$ & 145.4 & 10.5 & 0.0 & 87 \\
\hline
\end{tabular}


TABLE 1 - Continued

\begin{tabular}{|c|c|c|c|c|}
\hline $\begin{array}{l}\text { Core-Section, } \\
\text { Top of Interval } \\
\text { (cm) }\end{array}$ & $\begin{array}{l}\text { Subbottom } \\
\text { Depth } \\
(\mathrm{m})\end{array}$ & $\begin{array}{c}\text { Total } \\
\text { Carbon } \\
(\%)\end{array}$ & $\begin{array}{l}\text { Organic } \\
\text { Carbon } \\
\end{array}$ & $\mathrm{CaCO}_{3}$ \\
\hline $16-6,65$ & 148.0 & 10.6 & 0.0 & 88 \\
\hline $17-1,88$ & 149.9 & 10.5 & 0.0 & 87 \\
\hline $18-3,46$ & 162.0 & 11.5 & 0.0 & 95 \\
\hline $18-5,20$ & 164.7 & 10.4 & 0.0 & 87 \\
\hline $19-2,72$ & 170.2 & 11.5 & 0.0 & 96 \\
\hline $19-4,66$ & 173.2 & 11.5 & 0.0 & 95 \\
\hline $19-6,90$ & 176.4 & 11.2 & 0.0 & 93 \\
\hline $20-2,75$ & 179.8 & 11.8 & 0.0 & 98 \\
\hline $20-4,80$ & 182.8 & 11.8 & 0.0 & 98 \\
\hline $20-6,26$ & 185.3 & 11.8 & 0.0 & 98 \\
\hline $22-1,128$ & 197.8 & 11.7 & 0.0 & 97 \\
\hline $22-3,55$ & 200.1 & 11.8 & 0.0 & 98 \\
\hline $22-5,70$ & 203.2 & 11.7 & 0.0 & 97 \\
\hline $23-1,76$ & 207.3 & 11.7 & 0.0 & 97 \\
\hline $23-3,98$ & 210.5 & 11.7 & 0.0 & 97 \\
\hline $23-5,27$ & 212.8 & 11.5 & 0.0 & 95 \\
\hline $24-1,51$ & 216.0 & 11.7 & 0.0 & 97 \\
\hline $24-3,44$ & 218.9 & 11.6 & 0.0 & 96 \\
\hline $24-4,77$ & 220.8 & 11.7 & 0.0 & 97 \\
\hline $24-6,46$ & 223.5 & 11.8 & 0.0 & 98 \\
\hline $25-2,84$ & 227.3 & 11.8 & 0.0 & 98 \\
\hline $25-4,71$ & 230.2 & 11.8 & 0.0 & 98 \\
\hline $25-6,64$ & 233.1 & 11.8 & 0.0 & 98 \\
\hline $27-1,31$ & 244.3 & 11.7 & 0.0 & 97 \\
\hline $27-3,43$ & 247.4 & 11.5 & 0.0 & 95 \\
\hline $27-5,68$ & 250.7 & 11.8 & 0.0 & 98 \\
\hline $28-2,85$ & 255.9 & 11.8 & 0.0 & 98 \\
\hline $28-4,41$ & 258.4 & 11.8 & 0.1 & 98 \\
\hline $28-6,53$ & 261.5 & 11.9 & 0.0 & 98 \\
\hline $29-3,84$ & 266.8 & 12.0 & 0.0 & 99 \\
\hline $29-5,24$ & 269.2 & 12.0 & 0.0 & 100 \\
\hline $30-2,64$ & 274.6 & 11.8 & 0.0 & 98 \\
\hline $30-4,16$ & 277.2 & 11.8 & 0.0 & 98 \\
\hline $30-6,57$ & 280.6 & 11.8 & 0.0 & 98 \\
\hline $31-2,104$ & 284.5 & 11.8 & 0.0 & 98 \\
\hline $31-4,55$ & 287.6 & 12.1 & 0.0 & 100 \\
\hline $31-6,65$ & 290.7 & 11.7 & 0.0 & 97 \\
\hline $32-2,58$ & 293.6 & 11.8 & 0.0 & 98 \\
\hline $32-4,55$ & 296.6 & 11.9 & 0.0 & 99 \\
\hline $34-2,45$ & 312.5 & 12.0 & 0.0 & 99 \\
\hline $34-5,91$ & 317.4 & 12.0 & 0.1 & 100 \\
\hline $35-3,114$ & 324.1 & 12.0 & 0.2 & 99 \\
\hline $35-5,74$ & 326.7 & 11.9 & 0.0 & 99 \\
\hline $36-2,84$ & 332.3 & 12.0 & 0.0 & 100 \\
\hline $36-6,95$ & 338.5 & 11.5 & 0.0 & 95 \\
\hline $37-4,15$ & 343.7 & 11.6 & 0.0 & 96 \\
\hline $38-3,84$ & 352.3 & 11.9 & 0.0 & 99 \\
\hline $38-5,128$ & 355.8 & 11.9 & 0.0 & 98 \\
\hline $39-1,11$ & 358.6 & 11.7 & 0.0 & 97 \\
\hline $39-1,127$ & 359.8 & 11.9 & 0.0 & 99 \\
\hline $39-3,17$ & 361.7 & 11.8 & 0.1 & 98 \\
\hline $39-4,26$ & 363.3 & 12.1 & 0.0 & 100 \\
\hline $39-4,66$ & 363.7 & 11.6 & 0.0 & 96 \\
\hline $40-2,103$ & 370.0 & 11.6 & 0.0 & 96 \\
\hline $40-4,93$ & 372.9 & 12.0 & 0.0 & 100 \\
\hline \multicolumn{5}{|l|}{ Site 318} \\
\hline $1-1,126$ & 1.3 & 11.9 & 0.0 & 99 \\
\hline $1-2,82$ & 2.3 & 11.6 & 0.1 & 96 \\
\hline $1-3,70$ & 3.7 & 11.8 & 0.0 & 98 \\
\hline $1-4,81$ & 5.3 & 11.8 & 0.0 & 98 \\
\hline $1-5,98$ & 7.0 & 11.8 & 0.0 & 98 \\
\hline $2-2,30$ & 28.3 & 11.8 & 0.0 & 98 \\
\hline $3-1,46$ & 65.0 & 11.9 & 0.0 & 99 \\
\hline $4-1,49$ & 93.8 & 11.8 & 0.0 & 98 \\
\hline
\end{tabular}

TABLE 1 - Continued

\begin{tabular}{|c|c|c|c|c|}
\hline $\begin{array}{l}\text { Core-Section, } \\
\text { Top of Interval } \\
\text { (cm) }\end{array}$ & $\begin{array}{l}\text { Subbottom } \\
\text { Depth } \\
\text { (m) }\end{array}$ & $\begin{array}{c}\text { Total } \\
\text { Carbon } \\
(\%)\end{array}$ & $\begin{array}{c}\text { Organic } \\
\text { Carbon }\end{array}$ & $\mathrm{CaCO}_{3}$ \\
\hline $4-2,68$ & 95.5 & 11.7 & 0.0 & 97 \\
\hline $4-3,70$ & 97.7 & 11.5 & 0.0 & 96 \\
\hline $4-4,79$ & 98.6 & 11.8 & 0.0 & 98 \\
\hline $4-5,50$ & 99.8 & 11.7 & 0.0 & 97 \\
\hline $4-6,90$ & 101.7 & 11.8 & 0.0 & 98 \\
\hline $5-2,81$ & 124.0 & 12.0 & 0.0 & 100 \\
\hline $5-3,121$ & 125.9 & 12.0 & 0.0 & 99 \\
\hline $5-4,85$ & 127.1 & 11.9 & 0.0 & 99 \\
\hline $5-5,79$ & 128.5 & 11.8 & 0.0 & 98 \\
\hline $5-6,44$ & 129.6 & 11.2 & 0.1 & 92 \\
\hline $6-1,76$ & 151.3 & 11.6 & 0.0 & 96 \\
\hline $6-2,80$ & 152.8 & 11.9 & 0.0 & 99 \\
\hline $6-3,78$ & 154.3 & 12.0 & 0.1 & 100 \\
\hline $6-4,55$ & 155.6 & 12.0 & 0.1 & 100 \\
\hline $6-5,80$ & 157.3 & 12.0 & 0.0 & 100 \\
\hline $6-6,60$ & 158.6 & 12.0 & 0.0 & 100 \\
\hline $7-1,70$ & 179.7 & 11.9 & 0.0 & 99 \\
\hline $7-2,84$ & 181.3 & 11.5 & 0.0 & 96 \\
\hline $7-3,114$ & 183.1 & 11.8 & 0.0 & 98 \\
\hline $7-4,60$ & 184.1 & 11.6 & 0.0 & 96 \\
\hline $7-5,13$ & 185.5 & 11.7 & 0.0 & 97 \\
\hline $7-6,78$ & 187.3 & 11.7 & 0.0 & 98 \\
\hline $8-1,74$ & 207.7 & 11.8 & 0.0 & 98 \\
\hline $8-2,73$ & 209.2 & 11.8 & 0.0 & 98 \\
\hline $8-3,102$ & 211.0 & 11.7 & 0.0 & 97 \\
\hline $9-1,77$ & 236.3 & 11.9 & 0.0 & 99 \\
\hline $9-2,85$ & 237.9 & 11.9 & 0.0 & 99 \\
\hline $10-1,112$ & 265.1 & 0.6 & 0.1 & 4 \\
\hline $10-2,105$ & 266.6 & 11.4 & 0.0 & 95 \\
\hline $10-3,80$ & 267.8 & 11.6 & 0.0 & 96 \\
\hline $10-4,66$ & 269.2 & 11.4 & 0.0 & 94 \\
\hline $11-1,88$ & 293.4 & 11.3 & 0.0 & 94 \\
\hline $11-2,77$ & 294.8 & 11.6 & 0.0 & 96 \\
\hline $11-3,68$ & 296.2 & 11.8 & 0.0 & 98 \\
\hline $11-4,55$ & 297.6 & 11.5 & 0.0 & 95 \\
\hline $11-5,77$ & 299.3 & 10.6 & 0.0 & 88 \\
\hline $11-6,90$ & 300.9 & 11.7 & 0.0 & 97 \\
\hline $12-1,73$ & 321.7 & 11.6 & 0.0 & 97 \\
\hline $12-2,28$ & 322.8 & 11.2 & 0.0 & 93 \\
\hline $13-1,113$ & 350.6 & 11.8 & 0.0 & 98 \\
\hline $13-2,35$ & 351.4 & 11.4 & 0.0 & 94 \\
\hline $14-1,79$ & 378.8 & 10.9 & 0.0 & 91 \\
\hline $14-2,120$ & 380.7 & 11.0 & 0.0 & 91 \\
\hline $14-3,132$ & 382.3 & 11.2 & 0.0 & 93 \\
\hline $15-1,100$ & 407.5 & 11.6 & 0.0 & 96 \\
\hline $15-2,128$ & 409.3 & 11.0 & 0.0 & 91 \\
\hline $16-1,101$ & 436.0 & 11.8 & 0.0 & 98 \\
\hline $16-2,131$ & 437.8 & 11.7 & 0.0 & 97 \\
\hline $16-3,70$ & 438.7 & 11.7 & 0.1 & 97 \\
\hline $17-1,112$ & 464.6 & 11.6 & 0.0 & 96 \\
\hline $17-2,95$ & 466.0 & 11.6 & 0.1 & 96 \\
\hline $18-1,72$ & 492.7 & 11.6 & 0.1 & 96 \\
\hline $18-2,135$ & 494.9 & 11.9 & 0.1 & 98 \\
\hline $20-1,102$ & 550.0 & 11.3 & 0.1 & 93 \\
\hline $20-2,57$ & 551.1 & 11.4 & 0.1 & 94 \\
\hline $20-3,82$ & 552.8 & 11.5 & 0.1 & 95 \\
\hline $20-4,100$ & 554.5 & 11.4 & 0.1 & 95 \\
\hline $20-5,37$ & 555.4 & 6.7 & 0.1 & 55 \\
\hline $21-1,93$ & 578.4 & 11.1 & 0.1 & 92 \\
\hline $21-2,19$ & 579.2 & 10.6 & 0.1 & 87 \\
\hline $21-3,62$ & 581.1 & 10.3 & 0.4 & 83 \\
\hline $22-1,65$ & 597.2 & 10.0 & 0.1 & 83 \\
\hline $22-2,67$ & 598.7 & 10.4 & 0.1 & 86 \\
\hline $22-3,50$ & 600.0 & 4.6 & 0.1 & 37 \\
\hline $22-4,109$ & 602.1 & 9.6 & 0.1 & 80 \\
\hline $23-2,20$ & 607.7 & 9.7 & 0.2 & 79 \\
\hline $23-3,5$ & 609.1 & 6.8 & 0.4 & 54 \\
\hline
\end{tabular}


TABLE 1 - Continued

\begin{tabular}{lcccr}
\hline $\begin{array}{c}\text { Core-Section, } \\
\text { Top of Interval } \\
(\mathrm{cm})\end{array}$ & $\begin{array}{c}\text { Subbottom } \\
\text { Depth } \\
(\mathrm{m})\end{array}$ & $\begin{array}{c}\text { Total } \\
\text { Carbon } \\
(\%)\end{array}$ & $\begin{array}{c}\text { Organic } \\
\text { Carbon }\end{array}$ & $\mathrm{CaCO}_{3}$ \\
\hline $24-1,24$ & 615.7 & 2.2 & 0.1 & 17 \\
$24-2,26$ & 617.3 & 9.9 & 0.1 & 82 \\
$24-3,87$ & 619.4 & 9.9 & 0.1 & 82 \\
$24-4,73$ & 620.7 & 6.1 & 0.1 & 50 \\
$25-2,93$ & 627.4 & 9.4 & 0.1 & 78 \\
$25-3,20$ & 628.2 & 7.7 & 0.1 & 64 \\
$25-4,120$ & 630.7 & 0.7 & 0.1 & 5 \\
$26-2,36$ & 636.4 & 4.4 & 0.1 & 36 \\
$26-3,57$ & 638.1 & 0.7 & 0.1 & 5 \\
$26-4,83$ & 639.8 & 5.6 & 0.1 & 46 \\
$26-5,36$ & 640.9 & 0.9 & 0.1 & 7 \\
$27-1,107$ & 645.1 & 7.4 & 0.1 & 61 \\
$27-2,118$ & 646.7 & 5.9 & 0.1 & 48 \\
\hline
\end{tabular}

TABLE 1 - Continued

\begin{tabular}{lcccr}
\hline $\begin{array}{c}\text { Core-Section, } \\
\text { Top of Interval } \\
(\mathrm{cm})\end{array}$ & $\begin{array}{c}\text { Subbottom } \\
\text { Depth } \\
(\mathrm{m})\end{array}$ & $\begin{array}{c}\text { Total } \\
\text { Carbon } \\
(\%)\end{array}$ & $\begin{array}{c}\text { Organic } \\
\text { Carbon }\end{array}$ & $\mathrm{CaCO}_{3}$ \\
\hline $28-2,106$ & 665.6 & 0.5 & 0.1 & 4 \\
$28-3,102$ & 667.0 & 4.2 & 0.1 & 34 \\
$29-1,124$ & 683.2 & 1.2 & 0.2 & 9 \\
$29-2,93$ & 684.4 & 4.1 & 0.1 & 34 \\
$30-1,119$ & 702.2 & 0.4 & 0.2 & 2 \\
$30-2,132$ & 703.8 & 0.6 & 0.1 & 4 \\
$30-3,89$ & 704.9 & 5.3 & 0.1 & 43 \\
$31-2,47$ & 721.5 & 2.4 & 0.1 & 19 \\
$32-1,62$ & 739.6 & 0.3 & 0.1 & 2 \\
$32-3,69$ & 741.2 & 2.1 & 0.1 & 16 \\
$32-3,22$ & 742.2 & 3.1 & 0.1 & 24 \\
$32-4,3$ & 743.5 & 1.5 & 0.1 & 11 \\
& & & & \\
\hline
\end{tabular}

